\title{
PROGRESS IN THE DEVELOPMENT OF AN ELECTRO-MIGRATION MODEL- LING METHODOLOGY
}

\author{
Xiaoxin Zhu ${ }^{1}$, Hiren Kotadia ${ }^{2}$, Sha Xu ${ }^{3}$, Hua Lu ${ }^{1}$, Samjid. H. Mannan ${ }^{2}$, Chris Bailey ${ }^{1}$, Y.C Chan ${ }^{3}$ \\ ${ }^{1}$ School of Computing and Mathematical Sciences at University of Greenwich, 30 Park Row, London \\ SE10 9LS, UK (x.zhu@gre.ac.uk) \\ ${ }^{2}$ Physics Department, School of Natural \& Mathematical Sciences at King's College London, Strand, \\ London WC2R 2LS, UK
}

${ }^{3}$ Department of Electronic Engineering at City University of Hong Kong, 83 Tat Chee Avenue, Kowloon Tong, Hong Kong, China

\begin{abstract}
In this work, previous simulation works on electro-migration have been reviewed, and a multi-physics EM simulation method that combines electric, thermal, atomic diffusion and stress analysis has been proposed. The method is able to solve vacancy concentration distribution with coupling electric current, temperature gradient, hydro-static stress gradient simultaneously and to mimic void formation in realistic structure of microelectronics devices.
\end{abstract}

Keywords: Electro-migration, Thermo-migration, Stress-migration, Multi-Physics, Modelling.

\section{INTRODUCTION}

Electro-migration is the phenomenon of atomic migration under the influence of electric current, and is usually associated with thermal, and stress effects [1]. It is widely accepted that high current density is the main cause that increases the probability of the collision between electrons and diffusing atoms which result in void and hillock formation at the cathode and the anode respectively. In recent years, high density packaging (HDP) in electronics manufacturing has been increasingly adopted to meet the needs of miniaturization and increasing functionality in electronic products. Moreover, higher current density and complexity of structure has also generated high temperature gradients and high stress gradients leading to thermo-migration and stress-migration respectively in conductors. As a result, the true causes of EM involve a multi-physical cross coupling relationship and are poorly understood and characterized. In the past few decades, many researchers have studied EM and more recently computer simulation has become an ever more important tool in this research area. In this paper, previous works on EM simulation have been reviewed, and a new multi-physics EM simulation method that combines electric, thermal, atomic diffusion and stress analysis has been described. This new method is based on the multi-physics software PHYSICA and the 
aim of the work is to simulate the EM evolution process in interconnects in a unified environment and to help engineers achieve EM-aware electronics designs efficiently.

\section{PREVIOUS STUDIES REVIEW}

In 1960s, Black proposed a very simple model which first approaches the expression for the mean time to failure (MTTF) of a metal line subjected to electro-migration. He considered the MTTF is inversely proportional to the product of density of conducting electrons $\left(n_{e}\right)$, the momentum transfer from the electrons to the ions $(\Delta p)$, and the density of thermally activated ions $\left(N_{a}\right)$ as Eq. (1).

$$
M T T F \propto \frac{1}{n_{e} \Delta p N_{a}}
$$

Furthermore, he assumed that both the electron density as well as the momentum transferring is proportional to the current density $j$.

$$
n_{e} \propto j, \quad \Delta p \propto j
$$

And following the Arrhenius equation, we have Eq. (3).

$$
N_{a} \propto \exp \left(-E_{a} / k T\right)
$$

Rearranging equations, we can get Eq. (4).

$$
M T T F=\frac{A}{j^{2}} \exp \left(\frac{E_{a}}{k T}\right)
$$

where A is a material and geometry dependent constant [2 - 4], $E_{a}$ is the activation energy, $T$ is the temperature, and $k$ is Boltzmann's constant. However, many observations found that not all experimental observation match Eq. (4), but they could be matched by amending the current density exponent. Therefore, Black's equation was modified to Eq. (5).

$$
M T T F=\frac{A}{j^{n}} \exp \left(\frac{E_{a}}{k T}\right)
$$

Many studies have been carried out to determine the value of $n$. A study on bulk metals suggested that a value of $n=1$ is more appropriate [5]. D'Heurle and Agarwala [6, 7], through a series of experimental works, fitted the $n$ between 2 and 3 [8]. For a long time, there has been no theoretically trusted value, and some of previous experiments were challenged with regards to their thermal management. When a test is run with relatively low current density stressing and with the thermal effects decoupled, $n$ is reportedly between 1 and 2, but a value of 2 is probably used most widely [9].

Thermo-migration (TM) is almost inextricably linked to EM In addition, the composition of solder materials is moving towards lead-free Sn-based alloys that necessitate more investigation on the EM reliability issue due to poor field data availability [10]; Chih Chen et 
al, reported that the temperature gradient in a solder bump can reach $900-1000 \mathrm{~K} / \mathrm{cm}$ with the diameter of a flip-chip solder bump being reduced to $25 \mu \mathrm{m}[11,12]$ and that this temperature gradient is high enough to trigger thermo-migration (TM). A thermal analysis has been carried out on the software ANSYS and the result showed the contact site between $\mathrm{Cu}$ line and solder bump has the highest temperature is around $503 \mathrm{~K}$ (with ambient temperature $373 \mathrm{~K}$ ) and the temperature will significantly decrease to approximately $410 \mathrm{~K}$ because of the lower current density and the lager volume to dissipate heat in solder bump [12]. Chih Chen and et al. concluded that the $\mathrm{Al}$ trace generates most of the heat and therefore, the Al trace is the weakest site in the aspect of TM. A verification experiment was also carried out using an infrared microscopy. The results showed a SnAg solder bump with a 5- $\mu \mathrm{m} \mathrm{Cu} / 3-\mu \mathrm{m} \mathrm{Ni} \mathrm{UBM}$ can reach $2237 \mathrm{~K} / \mathrm{cm}$ with $0.6 \mathrm{~A}$, and the solder bump will likely melt with higher current stressing. After examining current density, Chih Chen et al also concluded that if the current density has a magnitude of $10^{4} \mathrm{~A} / \mathrm{cm}^{2}$ the driving force of EM is of the same magnitude as TM, but the driving force of TM will rise significantly beyond the driving force of EM with further current density increase and might cause solder bump melting.

Moreover, Stress-migration (SM) is due to the development of large tensile and compressive stress region at cathode and anode respectively as a combined consequence of the atomic migration and also due to the different thermal expansion coefficients of a narrow metal line and passivation or inter-metal dielectric material [13]. Hydro-static stress is proved to be the main driving force for void nucleation; regions with the most concentrated hydrostatic tensile stress are assumed to be the most probable sites for void nucleation $[14,15]$. The hydro-static stress, $\sigma_{H S}$, was defined as the average of $\sigma_{x}, \sigma_{y}, \sigma_{z}$. The hydrostatic stress gradient was defined as the ratio of the difference between stress values of two consecutives nodes to the distance between the nodes. The magnitude of the hydrostatic stress gradient $\left|\nabla \sigma_{H S}\right|$ is defined as $\sqrt{\left(\frac{\partial \sigma_{H S}}{\partial x}\right)^{2}+\left(\frac{\partial \sigma_{H S}}{\partial y}\right)^{2}+\left(\frac{\partial \sigma_{H S}}{\partial z}\right)^{2}}$.

Many researchers have attempted to model the growth of voids based on the SM studies in line structures with varying degrees of success over the years. Sauter et al [16] has developed a model, from which the time to failure, $t_{f}$, prediction due to void growth was found to follow the simple relation

$$
\frac{t_{f} \sigma^{2}}{d}=10^{19.2} \exp \left({ }^{Q} / R T\right)
$$

where $\sigma$ is the stress in the line, $d$ is the grain size, $Q$ is the activation energy for grain boundary diffusion in aluminum, $R$ is the universal gas constant, and $T$ is the absolute temperature at test. The stress $\sigma$ is given by

$$
\sigma=0.26(3) B \Delta \alpha \Delta T
$$

Where $B$ is the bulk modulus of Aluminum, $\Delta \alpha$ is the difference in thermal expansion coefficient between the line and the substrate and $\Delta T$ is the difference between the dielectric deposition temperature and the test temperature. P. M. Igic [17] proposed a method for calculating the thermal stress in aluminum interconnections during processing of the multilevel structure. 
Compared to previous studies [18], this technique allows the residual stresses from one processing step to be used as the initial conditions for a subsequent step by using a so called activating-deactivating strategy.

Since EM is caused by atomic/vacancy migration under the influence of electric current, convective diffusion algorithms can be employed to describe the EM process. Based on Fick's laws of diffusion, the governing equation for EM equation can be written as Eq. (8).

$$
\frac{\partial C_{v}}{\partial t}+\nabla \cdot J_{v}=r
$$

where $C_{v}$ is the vacancy concentration, $J_{v}$ is the total vacancy flux, $r$ is the source/sink term. $\mathrm{H}$ owever, in many EM works, atomic concentration $C_{a}$, rather than vacancy concentration $C_{v}$ was being used for simulating atomic movement. Because of the vacancy exchange mechanis $\mathrm{m}$, at equilibrium, the relation between the vacancy diffusivity and atomic diffusivity is given by [23]:

$$
C_{a} D_{a}=C_{v} D_{v}
$$

where $D_{a}$ is the diffusivity of atom. By using the Eq. (8), Kirsten and et al. [19] carried out a thermo-electrical-mechanical simulation of EM using ANSYS. The atomic flux from mechanical effect was also investigated but the atomic self-diffusion was not taken into account. The mass flux as well as mass flux divergence calculated. A steady state analysis was also implemented in this work to predict highest vacancy concentration site (weakest part of structure). Another similar computer simulation on EM for solder joint was proposed by Yong Liu et al [20-22]. In the simulation, elements with high vacancy concentration were 'killed' automatically to simulate void formation. F. Cacho et al [23] used the software package COMSOL to simulate atomic self-diffusion and stress evolution in 3D and 2D structures and predicted their MTTF for pre-set failure criterion in the terms of vacancy concentration. The failure criterion is arbitrary and moreover, the models could not select "dead elements" automatically.

\section{MODELLING}

In most computer simulation research work that has been done to date, not all of the physical processes which affect EM have been factored and this makes it difficult to compare simulation and experimental results. In this work, a closely coupled multi-physics modelling method has been proposed. It can be used to predict atomic concentration and void formation in metals where electrical, thermal, stress, and geometry factors are all taken into account. The schematic diagram Fig. 1 describes the methodology. The method has been implemented using the multi-physics software package PHYSICA [24], which is capable of solving fluid flow, heat transfer, electric field and general diffusion equations simultaneously. 
- Set initial parametersup

- Current Density, Temperature, Temperature Gradient, Hydro-

Step 3 static Stress and Hydro-static Stress Gradient Distribution

Step 4 - Calculate Vacancy distribution $\left(C_{v}\right)$

- Void Criterion? (True $>>$ Step 6 or False $>>$ Step 2)

- Set the related elements null

- Failure Judgement? (True >> Step 8 or False >> Step 2)

Figure 1: Flow Chart of the EM model

\subsection{GOVERNING EQUATIONS}

The governing diffusion-convection equation for vacancy evolution is Eq. (8). The total atomic flux can be described as Eq. (10).

$$
\begin{aligned}
& \boldsymbol{J}_{\mathrm{v}}=\boldsymbol{J}_{\text {diff }}+\boldsymbol{J}_{e m}+\boldsymbol{J}_{t h}+\boldsymbol{J}_{S} \\
& =-D_{\mathrm{v}}\left(\nabla C_{\mathrm{v}}-\frac{\left|Z^{*}\right| C_{v} \rho e}{k T} \boldsymbol{J}_{e}-\frac{Q^{*}}{k T^{2}} C_{\mathrm{v}} \nabla T+\frac{f \Omega}{k T} C_{\mathrm{v}} \nabla \sigma_{H S}\right)
\end{aligned}
$$

where

$$
D_{\mathrm{v}}=D_{\mathrm{v} 0} \exp \left(-\frac{E_{a}}{k T}\right)
$$

and $\boldsymbol{J}_{\text {diff }}$ is the vacancy flux caused by self-diffusion, $\boldsymbol{J}_{e m}$ is the flux caused by electric current effects, $\boldsymbol{J}_{t h}$ is the vacancy flux caused by thermal effects, $\boldsymbol{J}_{s}$ is the vacancy flux caused by stress effects, $D_{\mathrm{v}}$ is the diffusivity of atoms, $Z^{*}$ is the effective charge, $e$ is the elementary charge, $k$ is the Boltzmann's constant, $T$ is the temperature, $E$ is the electrical field, $Q^{*}$ is the heat of transport, $f$ is the vacancy relaxation factor, $\Omega$ is the atomic volume, $\sigma_{H S}$ is the hydrostatic stress, $D_{\mathrm{v} 0}$ is the pre-exponential factor and $E_{a}$ is the activation energy. The atomic flux $J_{\mathrm{a}}=-\boldsymbol{J}_{\mathrm{v}}$. 


\subsection{MODEL VALIDATION}

To test the modeling framework, a known 1D analytic solution of Eq. (8) and Eq. (10) (without the thermal and stress terms) as detailed by R.L. de Orio and his colleagues [25] was compared with the simulation results. The boundary conditions are shown in Eq. (12) which states that perfect blocking boundary conditions are imposed at both ends of the 1D domain.

$$
J_{v}(0, t)=J_{v}(-L, t)=0
$$

where $L$ is the length of the line.

$$
\frac{C_{v}(x, t)}{C_{v 0}}=A_{0}-\sum_{n=1}^{-\infty} A_{n} \exp \left(-B_{n} \frac{D_{v 0}}{L^{2}} t+\frac{\alpha}{2} \frac{x}{L}\right)
$$

where

and

$$
\alpha=\frac{\left|Z^{*}\right| e \rho j L}{k T}
$$

and

$$
\begin{gathered}
A_{0}=\frac{\alpha}{1-\exp (-\alpha)} \exp \left(\alpha \frac{x}{L}\right), \\
A_{n}=\frac{16 \pi \alpha^{2}\left[1-(-1)^{n} \exp \left(\frac{\alpha}{2}\right)\right)}{\left[\alpha^{2}+4 n^{2} \pi^{2}\right)^{2}}\left[\sin \left(n \pi \frac{x}{L}\right)+\frac{2 n \pi}{\alpha} \cos \left(n \pi \frac{x}{L}\right)\right]
\end{gathered}
$$

$$
B_{n}=n^{2} \pi^{2}+\alpha^{2} / 4
$$

Table I: Parameters used in the calculations

\begin{tabular}{|l|l|l|}
\hline Parameter & Value & Reference \\
\hline$D_{v 0}$ & $0.052 \mathrm{~cm}^{2} / \mathrm{s}$ & {$[27]$} \\
\hline$E_{a}$ & $0.9 \mathrm{eV}$ & {$[28]$} \\
\hline$Z^{*}$ & -5.0 & {$[29]$} \\
\hline$\rho$ & $1.69 \times 10^{-8} \Omega \mathrm{m}$ & {$[27]$} \\
\hline$j$ & $2 \mathrm{MA} / \mathrm{cm}^{2}$ & - \\
\hline$L$ & $100 \mu \mathrm{m}$ & - \\
\hline$T$ & $573 \mathrm{~K}$ & - \\
\hline
\end{tabular}

In the numerical simulation of this $1 \mathrm{D}$ problem, the domain has a length of $100 \mu \mathrm{m}$ and it was divided into 20 elements with constant cross-section area. An electric potential difference was applied at the two ends of the domain generating a uniform electric filed and a constant current. The parameters in Table I are used. The EM drift velocity is $0.214 \mathrm{~m} / \mathrm{s}$ if temperature or stress gradients are not taken into account. Fig. 2 shows that the analytical and simulation results for the vacancy concentration $\left(C_{\vee} / C_{v 0}\right)$ distribution are almost identical. 


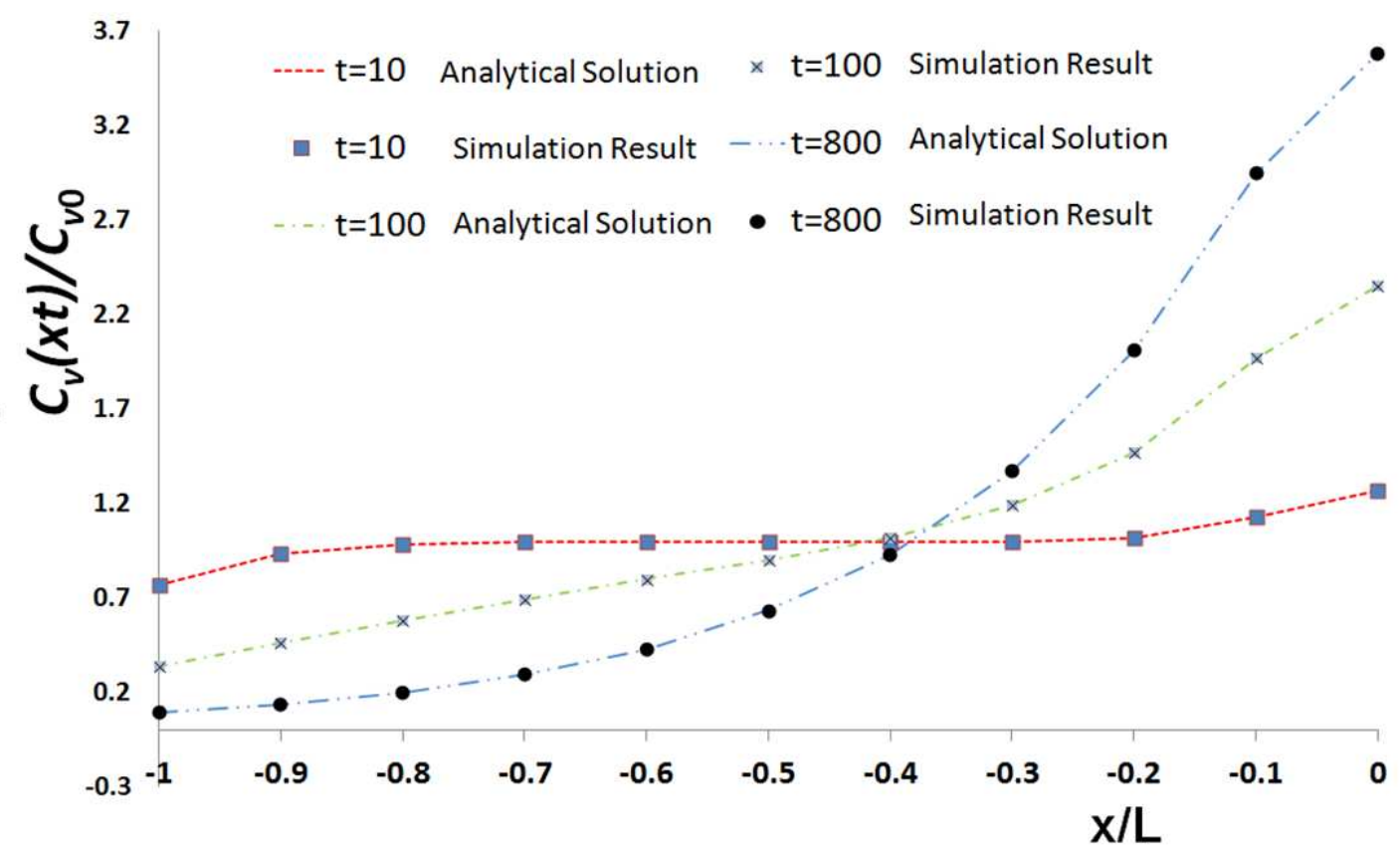

Figure 2: The analytical and simulation results for the vacancy concentration $\left(C_{\downarrow} / C_{v 0}\right)$ along the $\mathrm{X}$-axis at different times

In order to test the effect of temperature and stress gradients on EM, linear temperature and stress profiles were imposed. The values of the temperature and stress gradients are $0.107 \mathrm{~K} / \mathrm{m}$ and $0.107 \mathrm{MPa} / \mathrm{m}$ respectively. In Figure 3, Vacancy distributions at $\mathrm{t}=800 \mathrm{~s}$ are shown to be affected by temperature and stress gradients.

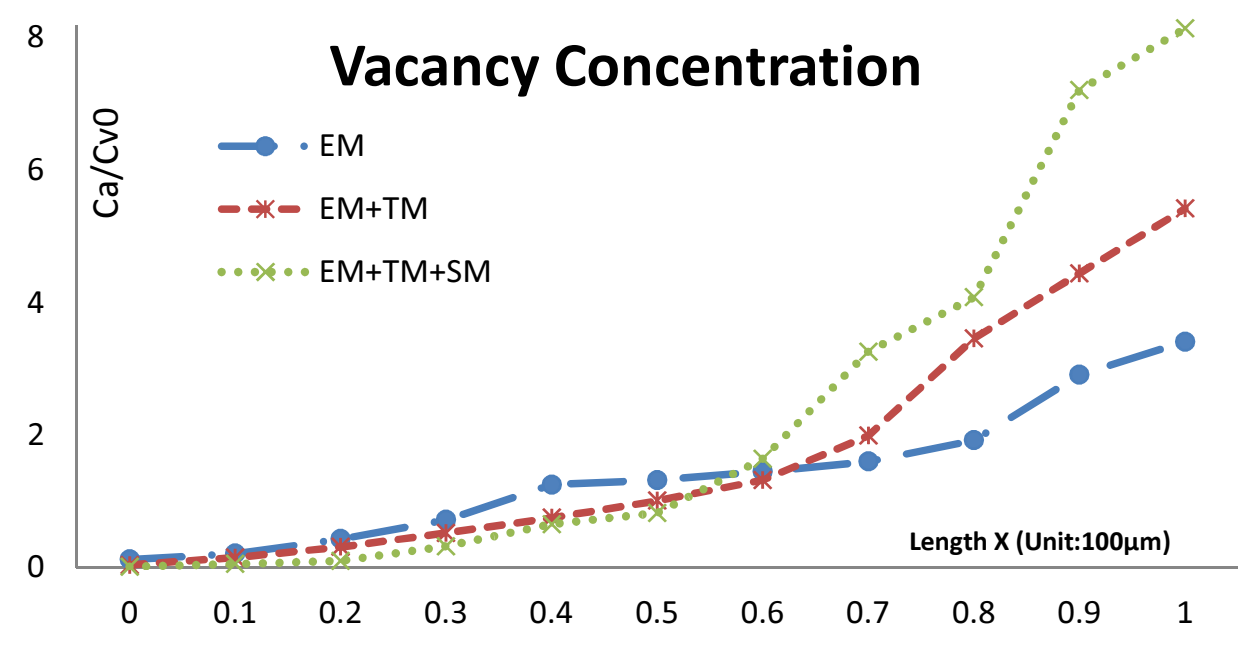

Figure 3: The vacancy concentration at $\mathrm{t}=800 \mathrm{~s}$.

\subsection{BLECH STRUCTURE}

The Blech structure (Fig. 4) has been modelled using the methods described above. The parameters in Table II were used in the modelling and a voltage load of $0.3 \mathrm{~V}$ was ap- 
plied. The ambient temperature was assumed to be $295 \mathrm{~K}$. The resulting current density, temperature and hydro-static stress distributions are shown in Fig. 5. The highest current density is about $6.18 \times 10^{5} \mathrm{~A} / \mathrm{cm}^{2}$. The maximum temperature is $456 \mathrm{~K}$ and it's located at the centres of tungsten parts. The highest hydrostatic stress magnitude is about $101 \mathrm{MPa}$ which appears at the W/Al interfaces. The vacancy flux divergence $\nabla \cdot J_{v}$ due to electro-migration, thermomigration and stress-migration are shown in Table III and Fig. 6.

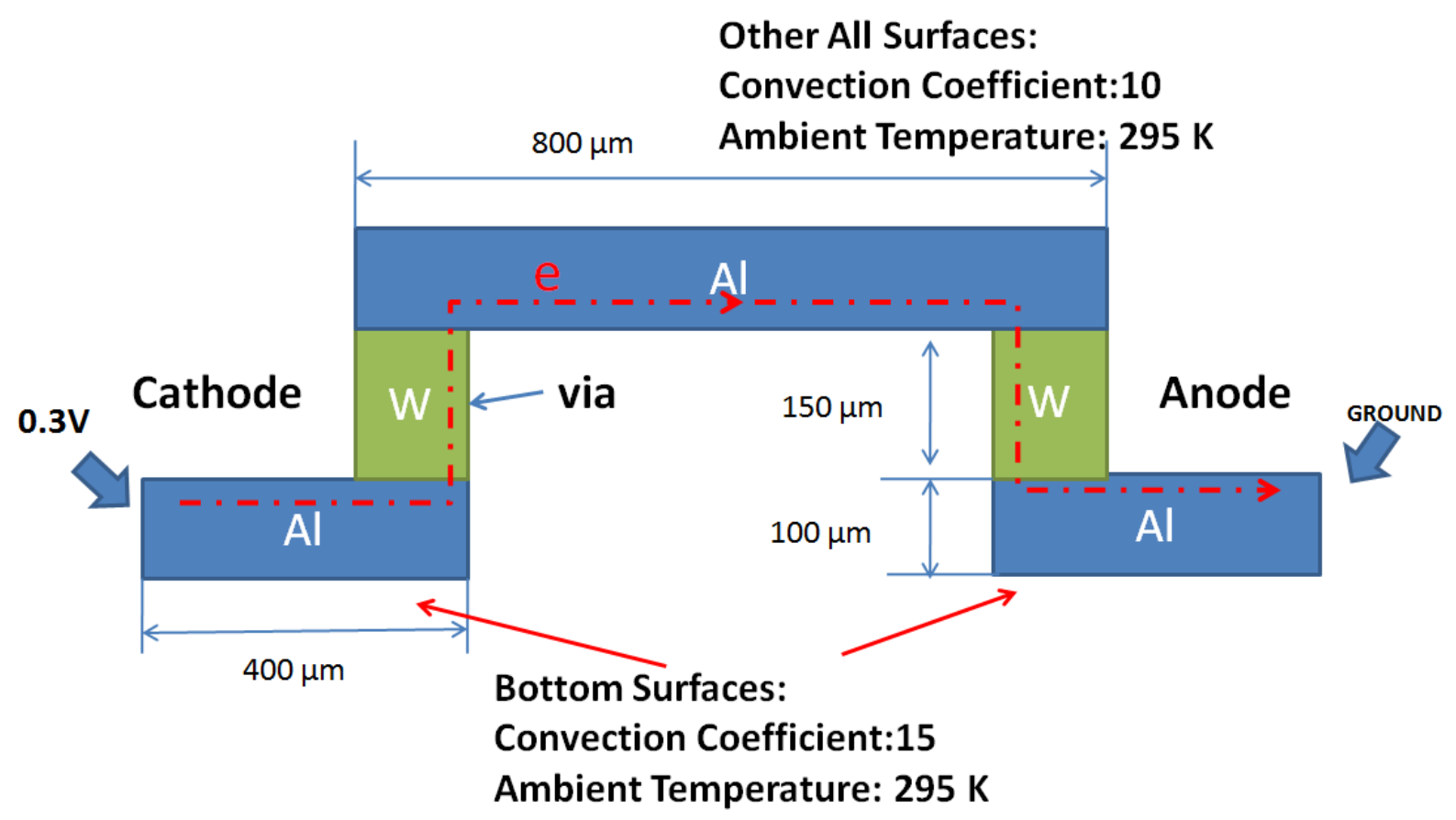

Figure 4 the schematic diagram of a Blech model and boundary conditions in the numerical simulation.

Table II: Parameters used in Blech Structure

\begin{tabular}{|l|l|l|l|}
\hline Parameter & Aluminum & Tungsten & Reference \\
\hline$D_{v 0}$ & $5.2 \times 10^{-2} \mathrm{~cm}^{2} / \mathrm{s}$ & $5.2 \times 10^{-4} \mathrm{~cm}^{2} / \mathrm{s}$ & {$[27]$} \\
\hline Activation Energy $(\mathrm{Ea})$ & $0.9 \mathrm{eV}$ & $1 \mathrm{eV}$ & Estimated \\
\hline$Z^{*}$ & -30.0 & -20.0 & Estimated \\
\hline$Q^{*}$ & $0.00094 \mathrm{eV}$ & $0.00094 \mathrm{eV}$ & {$[30]$} \\
\hline Atomic Volume $(\Omega)$ & $2.48 \times 10^{-29} \mathrm{~m}^{3} /$ atom & $2.48 \times 10^{-29} \mathrm{~m}^{3} /$ atom & {$[30]$} \\
\hline Resistivity $(\rho)$ & $2.82 \times 10^{-8} \Omega^{*} \mathrm{~m}$ & $5.60 \times 10^{-8} \Omega^{*} \mathrm{~m}$ & {$[31]$} \\
\hline Thermal Conductivity & $250 \mathrm{~W} / \mathrm{m}^{*} \mathrm{~K}$ & $173 \mathrm{~W} / \mathrm{m}^{*} \mathrm{~K}$ & {$[32]$} \\
\hline Specific Heat & $870 \mathrm{~J} / \mathrm{Kg}^{*} \mathrm{~K}$ & $170 \mathrm{~J} / \mathrm{Kg}^{*} \mathrm{~K}$ & {$[32]$} \\
\hline $\begin{array}{l}\text { Thermal Expansion Coef- } \\
\text { ficient }\end{array}$ & $22.2 \times 10^{-6} / \mathrm{K}$ & $4.3 \times 10^{-6} / \mathrm{K}$ & {$[32]$} \\
\hline Density & $2.7 \mathrm{~g} / \mathrm{cm}^{3}$ & $19.25 \mathrm{~g} / \mathrm{cm}^{3}$ & {$[32]$} \\
\hline Young's Modulus & $69 \mathrm{GPa}$ & $410 \mathrm{GPa}$ & {$[32]$} \\
\hline Poisson's Ratio & 0.334 & 0.284 & {$[32]$} \\
\hline
\end{tabular}




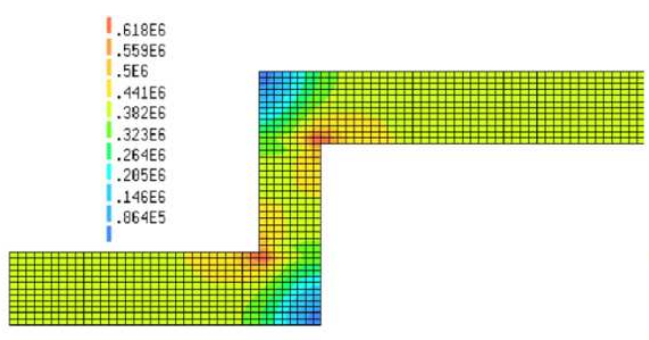

(a)

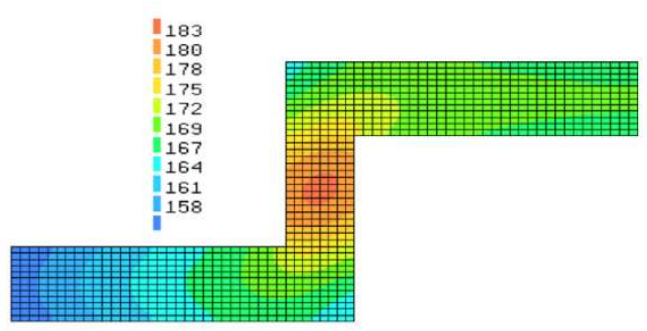

(b)

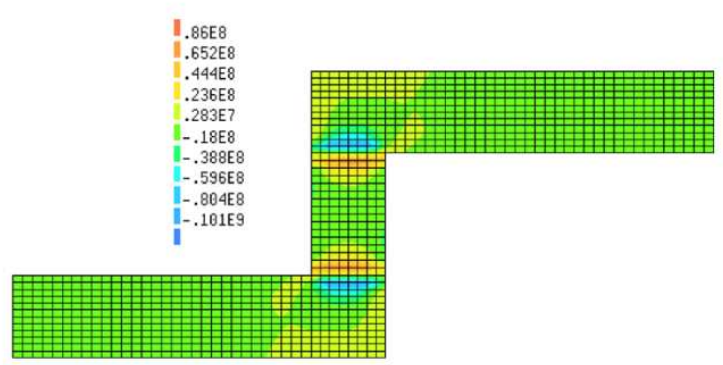

(c)

Figure 5 (a) Current density distribution, (b) Temperature distribution, and (c) Hydro-static stress distribution of Blech model

From Table III, we found that the maximum vacancy flux divergence of EM $\left(\boldsymbol{J}_{\text {em }}\right)$ is significantly greater then TM $\left(\boldsymbol{J}_{\boldsymbol{t} \boldsymbol{h}}\right)$ and SM $\left(\boldsymbol{J}_{\boldsymbol{s}}\right)$ and all maximum vacancy flux divergences are located around the interfaces of materials. We also noted that the maximum temperature gradient is just about $1570 \mathrm{~K} / \mathrm{cm}$ (Fig. 7). This value just reaches the criterion value of thermo-migration $[10,11,33]$. Therefore, at an ambient temperature of $295 \mathrm{~K}$ and in well ventilated conditions, the TM contributes the least to atomic migration compared to other effects on this structure.

Future work will be focused on the modeling of the combined effects of atomic migration in conductors and to model the void nucleation with the help of experiment to determine accurate activation energy $E_{a}$ and diffusion coefficient $D_{v}$, which significantly affect the modeling as discussed in our previous study [34].

Table III. Maximum atomic flux divergences due to EM, TM and SM with initial vacancy concentration $C_{v 0}=1$

\begin{tabular}{|c|c|c|c|c|}
\hline Divergence & Div $\boldsymbol{J}_{\text {em }}$ & Div $\boldsymbol{J}_{\text {th }}$ & Div $\boldsymbol{J}_{\boldsymbol{s}}$ & Unit \\
\hline Maximum & 0.188 & $0.431 \times 10^{-4}$ & $0.505 \times 10^{-1}$ & Atoms $/\left(\mathrm{m}^{3} \mathrm{~s}\right)$ \\
\hline Minimum & -0.188 & $-0.1435 \times 10^{-3}$ & $-0.2668 \times 10^{-1}$ & Atoms $/\left(\mathrm{m}^{3} \mathrm{~s}\right)$ \\
\hline
\end{tabular}




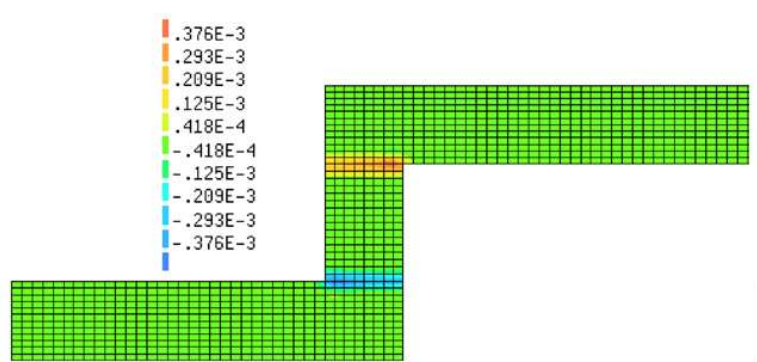

(a)

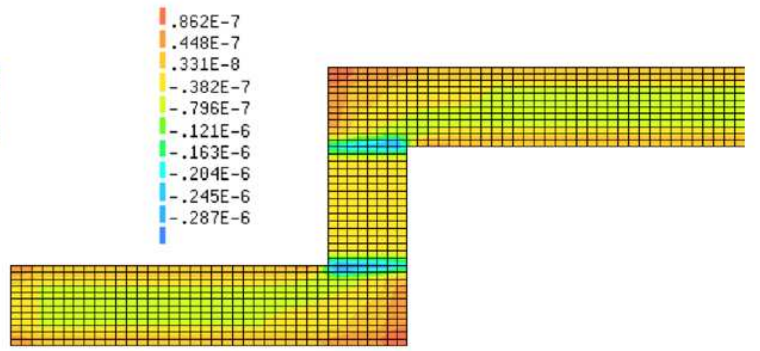

(b)

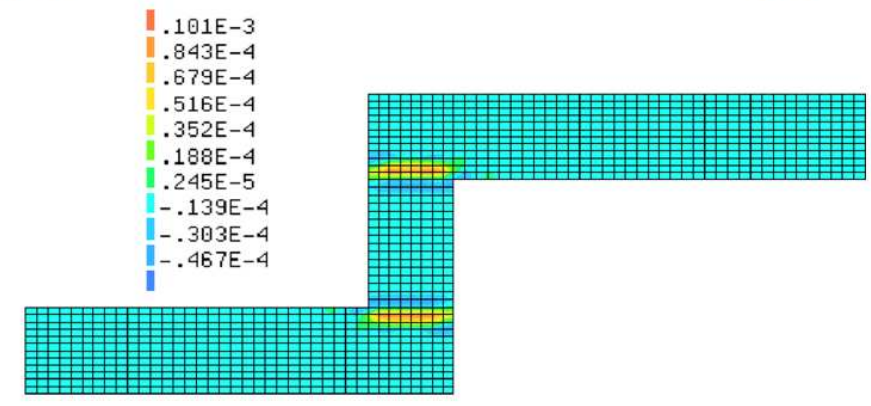

(c)

Figure 6: Snapshot of the distribution of divergence at $t=2000$ s for the (a) electrical, (b) thermal, and (c) stress vacancy flux.

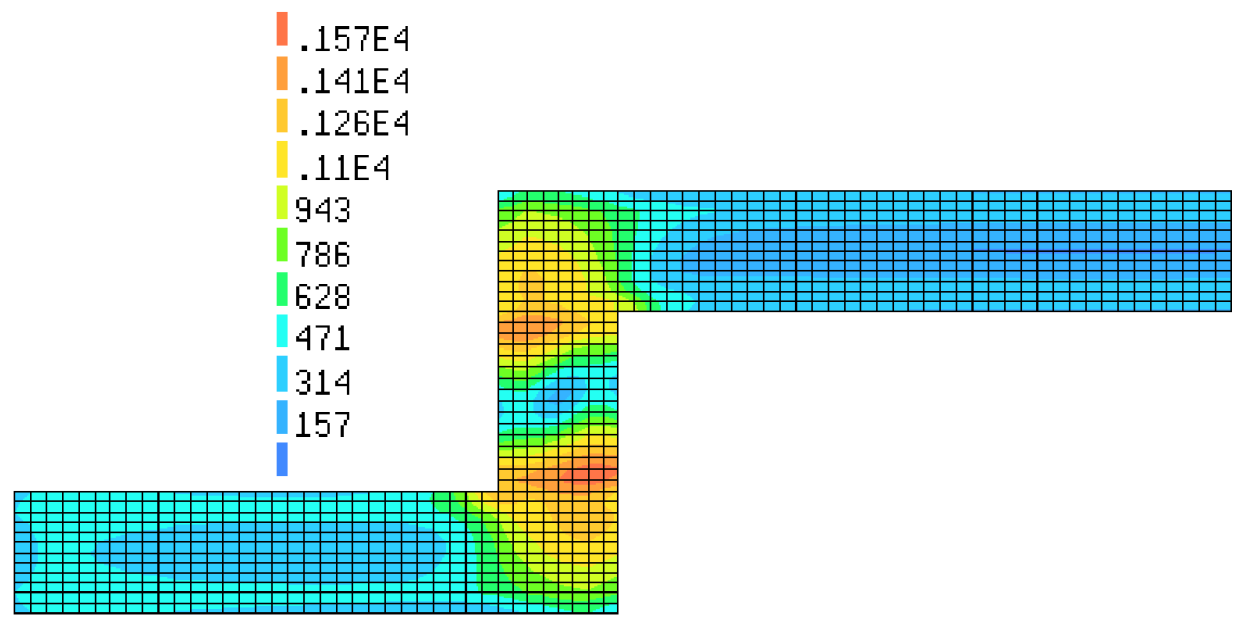

Figure 7: The temperature gradient distribution 


\section{CONCLUSION}

In this work, we reviewed the previous modelling work on EM and proposed a multiphysics modelling framework using the software package PHYSICA. The processes of diffusion, electric current, temperature gradient and stress gradient in electro-migration can be solved simultaneously. The computational model has been tested by a known analytical solution for a one dimensional problem and results are highly consistent with theoretical values. The Blech structure also has been implemented for comparing the contribution of individual effects.

\section{ACKNOWLEDGEMENTS}

Xiaoxin Zhu would like to thank the University of Greenwich and Sha Xu would like to thank HK RGC GRF (Project No.9041486), CityU Research Activity Funds for financing this research work.

\section{REFERENCE}

[1] King-Ning Tu, "Solder Joint Technology - Materials, Properties, and Reliability” Springer 2007.

[2] Black JR. Mass transport of aluminum by momentum exchange with conducting electrons. Proc 6th Ann Reliab Phys Symp 1967:148 - 59.

[3] Black JR. Electromigration - a brief survey and some recent results. IEEE Trans Electron Devices 1969;16(4):338 - 47.

[4] Black JR. Electromigration failure modes in aluminum metallization for semiconductor devices. Proc IEEE Lett 1969;57(9):1578 - 94.

[5] Huntington, H.B and Grone,A.R. ,J.Phys Chem. Solid, 20,76,(1961)

[6] D'Heurle, F.M., Metallurgical Transaction, 2,683 ( 1971)

[7] Agarwala, B.N. in Proc. 13th Int. Reliability Physics Symp, IEEE, 1975, p 107

[8] Schafft, H.A., Grant, T.C., Saxena, A. N., and Kao, C., in Proc. 23rd Int. Reliability Physics Symp., IEEE, 1985,. P93

[9] Lloyd JR. Black's law revisited - nucleation and growth in electromigration failure. Microelectron Reliab 2007; 47:1468-72.

[10] Zeng K, Tu KN. 2002. Six cases of reliability study of Pb-free solder joints in electronic packaging technology. Mater. Sci. Eng. Rep. 38:55-105

[11] Wright SL, Polastre R, Gan H, Buchwalter LP, Horton R, et al. 2006. Characterization of micro-bump C4 interconnects for Si-carrier SOP applications. Proc. Electron. Compon. Technol. Conf., 56th, San Diego15:633-40

[12] Chih Chen, H.M. Tong, and K.N. Tu, Electromigration and Thermomigration in PbFree Flip-Chip Solder Joints, Annu Rev. Mater, Res, 2010. 40: 531-555

[13] A. Mathewson, P.O'Sullivan et al, "Modelling and Simulations of Reliability for Design", Microelectronic Engineering 49 (1999), pp.95-117. 
[14] Yuan Guangjie and Chen Leng, "Finite Element Simulation of Hydrostatic Stress in Copper Interconnects", Journal of Semiconductors (2011), Vol.32, No.5.

[15] L. T. Shi and K. N. Tu, "Finite-element Modelling of Stress Distribution and Migration in Interconnecting Studs of a Three Dimensional Multillevel Device Structure", Applied Physics Letter 65 (12), Sep 1994

[16] A.I.Sauter and W.D.Nix, "Stress-Induced Phenomena in Metallization”, Journal of Materials Research 7 (1987) pp.1133-1325

[17] P. M. Igic, P. A. Mawby, "An Advanced Finite Element Strategy for Thermal Stress Field Investigation in Aluminium Interconnections during Processing of very Large Scale Integration Multilevel Structures", Microelectronics Journal 30 (1999), pp.1207-1212

[18] Y. L. Shen, "Thermo-Mechanical Stresses in Copper Interconnects - A Modelling Analysis", Microelectronic Engineering 83 (2006), pp.446-459

[19] Kirsten Weide-Zaage, David Dalleau et al., "Static and Dynamic Analysis of Failure Locations and Void Formation in Interconnects due to Various Migration Mechanisms", Material Science in Semiconductor Processing 6 (2003), pp.85-92.

[20] Yong Liu, Lihua Liang, Scott Irving, Timwah Luk, 3D Modeling of electromigration combined with thermal-mechanical effect for IC device and package, Microelectronics Reliability, 48 (2008) 811-824

[21] Liang LH, Xu YJ, Liu Y. Electro-migration study in solder joint and interconnects of IC packages. In: Proceedings of EuroSIME2006, April 2006, Como/Italy. p. 464-70.

[22] Liang LH, Liu Y. Reliability study in solder joint under electromigration thermalmechanical load. In: International conference on electronics packaging technology, ICEPT2006. p. 861

[23] F. Cacho, V.Fiori, C. Chappaz, C.Tavernier, H.Jaouen, Modeling of Electromigration Induced Failure Mechanism in Semiconductor Devices, Excerpt from the proceedings of the COMSOL Users Conference 2007 Grenoble

[24] Physica Ltd, 3 Rowan Drive, Witney, Oxon, United Kingdom, http://www.physica.co.uk/

[25] R.L. de Orio, H. Ceric, S. Selberherr, "Physically based models of electromigration: From Black's equation to modern TCAD models", P775-789, Microelecronics Reliability, 2010

[26] de Groot SR. “Theorie phenomenologique de L'Effet soret”. Physica 1942:699-707

[27] Sukharev V, Zschech E, Nix WD. "A model for electromigration-induceddegradation mechanisms in dual-inlaid copper interconnects: effect of microstructure." J Appl Phys 2007;102:053505.

[28] Liniger EG, Gignac LM, Hu C-K, Kaldor S. "In situ study of void growth kinetics in electroplated Cu lines". J Appl Phys 2002;92(4):1803-10.

[29] Lodder A, Dekker JP. "The electromigration force in metallic bulk". AIP Conf Proc 1998;418:315-29.

[30] Yong Liu, Qiang Wang, Lihua Liang, Xuefan Chen, Scott Irving and Timwah Luk, A New Prediction Methodology for Electromigration-Induced Solder Degradation in a WLCSP System, 2009 Electronic Components and Technology Conference

[31] Serway, Raymond A. (1998). Principles of Physics (2nd ed ed.). Fort Worth, Texas; London: Saunders College Pub. p. 602. ISBN 0-03-020457-7.

[32] Engineering Tool Box, http://www.engineeringtoolbox.com 
[33] Annie T. Huang and K.N. Tu, Effect of the combination of electromigration and thermomigration on phas migration and partial melting in flip chip composite $\mathrm{SnPb}$ solder joints, Journal of Applied Physics; 100, 033512 (2006)

[34] Xiaoxin Zhu, Hiren Kotadia, Sha Xu, Hua Lu, Samjid H. Mannan, YC Chan, Chris Bailey, Multi-physics Computer Simulation of the Electromigration Phenomenon, C-005, ICEPT-HDP 2011, IEEE, Shanghai, China 